\section{A significant breakthrough for caries management}

Using a specific photoprotein that produces bioluminescence in the presence of free calcium ions, the CALCIVIS system displays a visual map of active demineralisation at the chair side. This enables practitioners and their patients to clearly see the very early signs of caries

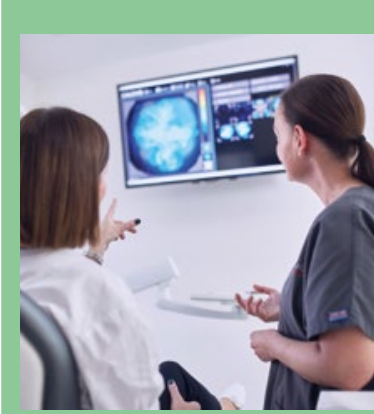
long before surgical intervention is required.

With definitive evidence of active demineralisation, preventive measures can be implemented to prevent the tooth progressing to the cavitation stages. By identifying free calcium ions so precisely, the CALCIVIS system provides a means of assessing the efficacy of reparative or remineralisation therapies.

To see and learn more about the new CALCIVIS system, visit www.calcivis.com, call 01316585152 or email info@ calcivis.com.

\title{
Beyond the call of duty
}

Seek out Clark Dental for professional services that go above and beyond the call of duty.

The expert team have developed an exceptional reputation as one of Britain's foremost dental equipment suppliers, offering a range of advanced solutions from leading brands such as A-dec, Anthos and Sirona.

As part of the all-inclusive service, Clark Dental also utilises the latest CAD technology and decades of experience to help practitioners design their dream surgery or complete practice. Clark Dental considers every detail of surgery design, of the design outfitting your surgery with bespoke dental cabinetry and state-of-the-art equipment to suit any budget as well as providing outstanding customer service.

For more information call Clark Dental on 01268733 146, email info@clarkdental.co.uk or visit www.clarkdental.co.uk.

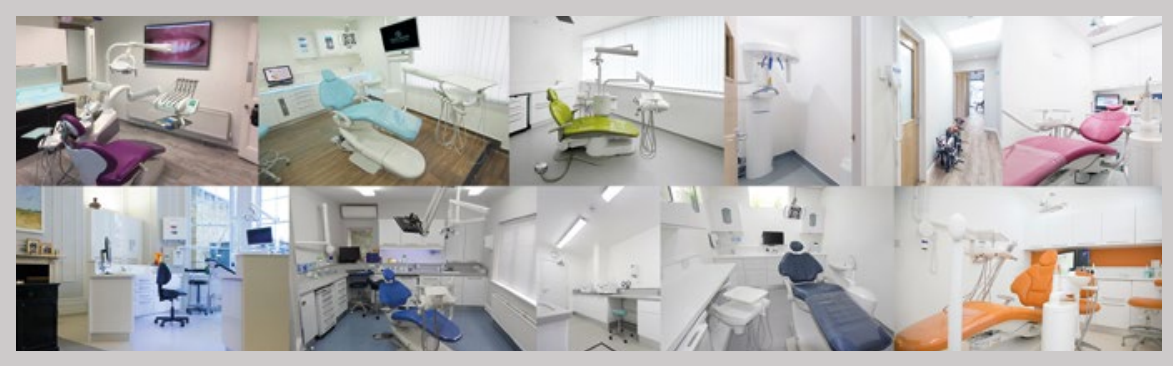

\section{Feeling fine}

Help patients avoid the risk of oral diseases with some of the finest interdental brushes on the market: leading oral healthcare specialist, Curaprox, offers you the CPS Prime range.

The high quality of these products including Prime Plus and Prime Handy - ensure that one movement in and out between each tooth is all it takes for safe and effective cleaning. This is thanks to the conical design of CPS Prime brushes, which feature nickel-free, $\mathrm{CURAL}^{\bullet}$ surgical wires that can

clean the smallest of interdental spaces without risk of injury.

Explore

the durability of CPS Prime interdental brushes by contacting Curaprox on 01480 862084, info@curaprox.co.uk or visiting www.curaprox.co.uk.

\section{Improving men's oral health}

Inspire more

men to see the benefits of good oral health, great looking teeth and fresh breath with CB12 White.

CB12 White mouthwash enhances the oral

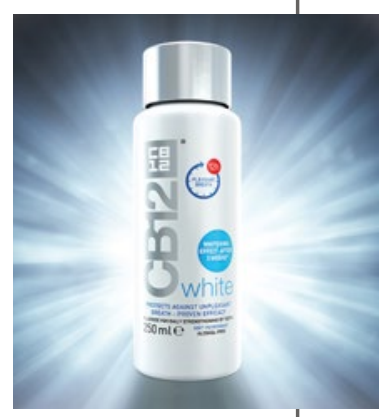

hygiene routine,

prevents oral malodour and brightens the teeth. As an adjunct to brushing and flossing, it helps to rinse away debris and bacteria and contains active ingredients that prevent unpleasant smelling breath for up to 12 hours.

It also has anti-plaque agents and fluoride to strengthen the teeth along with the technology to lift tooth stains and prevent new stains from developing. Yes, used twice a day for two weeks, CB12 White produces a pleasing whitening effect that patients are sure to find appealing.

For more information about CB12 and how it could benefit your patients, visit www.cb12.com.

\section{Offer your NHS patients more}

It's important to be able to supply your patients with a wide range of treatment options. So why not let them experience enhanced aesthetics with Monolith FullContour Zirconia from CosTech Dental Laboratory - the first zirconia restorations available on the NHS.

Priced at just $£ 29.95$ per unit, including free delivery to and from the laboratory, Monolith Full-Contour Zirconia allows patients to choose a strong and aesthetic restorative option. They're also completely metal-free!

For more information about CosTech Dental Laboratory visit www.costech. co.uk or call 01474320076. 